Cahiers Charlevoix

Cahiers Charlevoix

Études franco-ontariennes

\title{
Trois littératures francophones au Canada 1972-1992
}

\section{René Dionne}

Volume 3, 1998

URI : https://id.erudit.org/iderudit/1039396ar

DOI : https://doi.org/10.7202/1039396ar

Aller au sommaire du numéro

Éditeur(s)

Société Charlevoix

Presses de l'Université d'Ottawa

ISSN

1203-4371 (imprimé)

2371-6878 (numérique)

Découvrir la revue

Citer cet article

Dionne, R. (1998). Trois littératures francophones au Canada 1972-1992. Cahiers

Charlevoix, 3, 197-229. https://doi.org/10.7202/1039396ar

\section{Résumé de l'article}

René Dionne, qui embrasse dans un même parallèle « trois littératures francophones du Canada », évoque l'émergence de ces entités régionales du Canada français et leur développement récent, entre 1972 et 1992. Après leur affranchissement du Québec, qui avait confisqué et, à rebours, rebaptisé " québécois » tout l'héritage qui forma jadis la feue « littérature nationale » ou " canadienne ", puis naguère " canadienne-française ", ces trois littératures ont vécu des stades de contestation puis de récupération de leur patrimoine écrit. Le réveil des identités régionales, excité par l'attitude souvent méprisante du Québec, a provoqué la prise de parole des minorités qui, aidées de leurs établissements séculaires et des gouvernements, ont institué de plus en plus solidement et complètement les littératures acadienne, franco-ontarienne et francophone de l'Ouest.
Ce document est protégé par la loi sur le droit d'auteur. L'utilisation des services d'Érudit (y compris la reproduction) est assujettie à sa politique d'utilisation que vous pouvez consulter en ligne.

https://apropos.erudit.org/fr/usagers/politique-dutilisation/ 


\section{TROIS LITTÉRATURES}

fRancophones au Canada

\section{2-1992}

René Dionne

Département des lettres françaises

Université d'Ottawa

Cahiers Charlevoix 3, 1998, pp. 197-229. 


\section{SOMMAIRE}

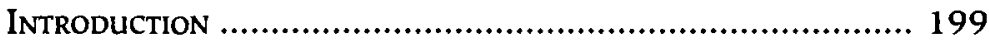

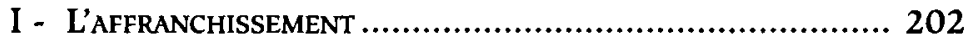

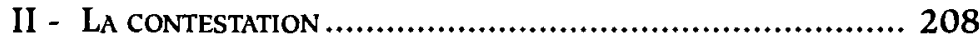

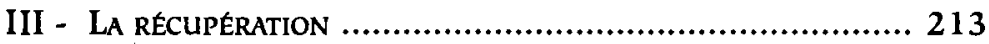

IV - Des institutions Nouvelles ou ReNouvelÉES ............... 217

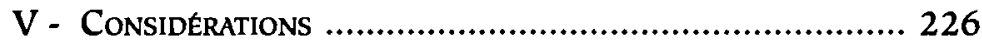




\section{TROIS LITTÉRATURES FRANCOPHONES AU CANADA 1972-1992}

\section{INTRODUCTION}

Durant plus d'un siècle, plus précisément des années 1830 à la décennie de 1960, il n'exista qu'une seule littérature de langue française au Canada; c'était une littérature dite nationale ${ }^{2}$ qui appartenait au même pays que la littérature canadienne-anglaise,

\footnotetext{
${ }^{1}$ Le texte qui suit est la deuxième version révisée et augmentée de celui qui a paru sous le même titre dans Mélanges Marguerite Maillet, recueil de textes de création et d'articles sur la littérature, la langue et l'ethnologie acadiennes en hommage à Marguerite Maillet, sous la direction de Raoul Boudreau, Anne Marie Robichaud, Zénon Chiasson et Pierre M. Gérin, Moncton, Chaire d'études acadiennes et Éditions d'Acadie, "Mouvance», 4, 1996, pp. 161-180.

${ }^{2}$ Exemples: Répertoire national ou Recueil de littérature canadienne, compilé et édité par James Huston, 4 vol., Montréal, de l'Imprimerie Lovell et Gibson, 1848, 1848, 1848 et 1850, viii, 368[8], 376[6], 384[2] et 404[4] p.; [Laurent-]O[livier] David, "Essai sur la littérature nationale", l'Écho du Cabinet de lecture paroissial, vol. 3, n ${ }^{\circ} 40,12$ octobre 1861, pp. 315-318, (voir notre texte, «Essai sur la littérature nationale (1861): un texte trop négligé», dans Prefaces and Literary Manifestoes / Préfaces et manifestes littéraires, edited by E.D. Blodgett and A.G. Purdy, in collaboration with S. Tötösy de Zepetnek, with the assistance of C. Garret and L.-A. Lavigne. Proceedings of a Conference.
} 
nationale elle aussi. La première était canadienne pour le francophone ${ }^{3}$ et la seconde, Canadian pour l'anglophone; ${ }^{4}$ on se partageait le qualificatif comme on se partageait le pays: entre deux langues. Il arriva aussi aux premiers historiens littéraires de réunir l'une et l'autre littératures sous la même étiquette 5 . Contrairement au Canada anglais qui, sous l'influence du One Country, One Nation, est resté fidèle à cette pratique jusqu'à tout récemment ${ }^{6}$, le Canada français s'en est détaché depuis belle lurette. Les historiens francophones ont même remplacé très tôt l'appellation canadienne par celle de canadienne-française ${ }^{7}$ et les anglophones ont parfois

[...], [Edmonton], Research Institute for Comparative Literature, University of Alberta, 1990, pp. 158-170); Henri-Raymond Casgrain, "Le Mouvement littéraire en Canada», Le Foyer canadien, vol. IV, 1866, pp. 1-31, (voir notre texte, «Le Mouvement littéraire en Canada, texte d'un homme ou d'une société?», dans l'Histoire littéraire. Théories, méthodes, pratiques, sous la direction de Clément Moisan, Québec, les Presses de l'Université Laval, 1989, pp. 271-280).

${ }^{3}$ Exemples: [Antoine Gérin-Lajoie, compilateur], La Littérature canadienne de 1850 a 1860, publiée par la direction du Foyer canadien, tome 1, Québec, Desbarats et Derbishire, 1863, 388 p.; [Antonin Nantel, compilateur], Les Fleurs de la poésie canadienne, Montréal, C. O. Beauchemin \& Valois, libraires-imprimeurs, 1869, 134 p.; Edmond Lareau, Histoire de la littérature canadienne, Montréal, imprimé par John Lovell, 1874, viii, 496 p.; Jules Léger, Le Canada et son expression littéraire, Paris, Librairie Nizet et Bastard, 1938, [iv], 211 [2] p.

${ }^{4}$ Exemple: Archibald MacMurchy, Handbook of Canadian Literature, 1906.

${ }^{5}$ Exemples: Henry J. Morgan, Bibliotheca Canadensis; or, A Manual of Canadian Literature, Ottawa, G. E. Desbarats, 1867, xiv, 411 p.; Archibald MacMechan, Headwaters of Canadian Literature, Toronto, McClelland \& Stewart, 1924, 247 p.; Lorne Pierce, An Outline of Canadian Literature (French and English), Toronto, The Ryerson Press, 1927, [xi], 251 p.; Camille Roy, Histoire de la littérature canadienne, nouvelle édition revue et mise à jour, Québec, Imprimerie de «l'Action sociale», 1930, $310 \mathrm{p}$.

${ }^{6}$ Exemple: Norah Story, The Oxford Companion to Canadian History and Literature, Toronto, Oxford University Press, 1967, xi, [ix], 935 p.

${ }^{7}$ Exemples: "Discours de M. Pamphile Lemay, sur la littérature 
utilisé le qualificatif English-Canadian ${ }^{8}$.

Mais à partir de la décennie de 1960, l'étiquette "canadienne-française» est honnie par les séparatistes du Québec: Québécois ils sont, québécoise sera dorénavant leur littérature comme la nation qu'ils forment. Des Québécois francophiles que cette appellation indispose parleront pendant un temps de littérature française du Québec ${ }^{9}$, tandis que maints fédéralistes plieront l'échine peu à peu devant l'ampleur d'un mouvement qui gagnera rapidement l'ensemble de la population de la province ${ }^{10}$ et poussera l'impérialisme linguistique jusqu'à qualifier de québécois ou "du Québec» tout ce qui bouge en français au Canada ${ }^{11}$. Aujourd'hui, le qualificatif canadien-français n'est plus guère utilisé que par des Canadiens non québécois; il arrive même à ces derniers de parler plutôt de littérature canadienne de langue française $^{12}$ ou encore de littérature canadienne

canadienne-française et sa mission", dans H.-J.-J.-B. Chouinard, La Fête des Canadiens-Français [sic] célébrée d Québec en 1880 [...], Québec, de l'Imprimerie A. Côté et $C^{\text {‘e }}$, 1881, pp. 375-383; Camille Roy, Tableau de l'histoire de la littérature canadienne-française, Québec, l'Imprimerie de "l'Action sociale», 1907, 81[2] p.

${ }^{8}$ Exemple: Thomas G. Marquis, English-Canadian Literature, 1914.

${ }^{9}$ Exemple: Pierre de Grandpré, (dir.), Histoire de la littérature française du Québec, 4 tomes, Montréal, Librairie Beauchemin, 1967, 1968, 1969, 1969, 368, 390, 407, 428 p.

${ }^{10}$ Exemple: Gilles Marcotte (dir.), Anthologie de la littérature québécoise, Montréal, La Presse, 1978-1980, 4 vol.; [2 ${ }^{\mathrm{c}}$ éd. révisée], Montréal, L'Hexagone, 1994, $2 \mathrm{t}$.

${ }^{11}$ Exemple: Maurice Lemire (dir.), Dictionnaire des ceuvres littéraires du Québec, Montréal, Fides, 1978-1987, 5 tomes. [Le cinquième tome (1987) essaie d'éviter cet impérialisme; voir "Avertissement», p. lxxi.] Un sixième tome a paru en 1994 sous la direction de Gilles Dorion.

${ }^{12}$ Exemple: René Dionne, La Littérature canadienne de langue française/ Canadian Literature in French, Ottawa, Secrétariat d'État du Canada/ Department of the Secretary of State of Canada, «Guide pédagogique des études canadiennes/Canadian Studies Resource Guides», 1988, $35 / 31 \mathrm{p}$. 
francophone ${ }^{13}$ lorsqu'ils considèrent les œuvres écrites et publiées en français sur l'ensemble du territoire canadien, ce qui a amené des Canadiens anglais à écrire en parallèle Canadian Literature in English ${ }^{14}$, qui n'avait servi que de sous-titre à la Literary History of Canada ${ }^{15}$ de Carl F. Klinck.

\section{I - L'AFFRANChisSEMENT}

Question de mots que tout cela? Non, problème d'une identité à choisir et à définir. Le million de francophones canadiens qui vivent dans une autre province que le Québec ne veulent pas s'appeler Québécois, car ils ne le sont pas, ne le sont plus ou ne l'ont jamais été. La moitié d'entre eux ont des origines québécoises plus ou moins lointaines: ils habitent principalement l'Ontario et les provinces de l'Ouest, et chacune de ces provinces leur est un pays aussi cher que le Québec peut l'être à ses habitants. Les francophones des Maritimes, qui comptent pour un tiers de la population francophone du Canada, sont en grande partie d'origine française, et c'est sous des régimes particuliers ou différents de celui des Québécois qu'ils ont vécu les deux premiers siècles et demi de leur existence collective dans une Acadie qu'ils considèrent comme leur pays, même si elle est aujourd'hui partagée principalement entre trois provinces. Quant aux autres francophones cana-

\footnotetext{
${ }^{13}$ Exemple: Edwin Hamblet, La Littérature canadienne francophone, Paris, Hatier, "Profil formation: français", 419-420, 1987, 159 p.

${ }^{14}$ Exemple: Bruce Nesbitt, Canadian Literature in English/la Littérature canadienne de langue anglaise, Ottawa, Department of the Secretary of State of Canada/Secrétariat d'État du Canada, "Canadian Studies Resource Guides/Guide pédagogique des études canadiennes», 1988, $41 / 44$ p.

${ }^{15}$ Literary History of Canada: Canadian Literature in English, Toronto, University of Toronto Press, 1965, xiv, 945 p.
} 
diens d'origines diverses, européennes surtout, seule l'appellation de Canadiens français les rattachait au Québec, foyer central de ce pays linguistique qu'était le Canada français. Le Québec se séparant, ce million de francophones perd son point d'appui traditionnel; et l'appellation «francophones hors Québec», que l'on utilisera jusqu'en 1991 pour désigner ces Canadiens, exprime bien le rejet dont ils sont l'objet de la part de la province mère, naturelle ou adoptive selon les cas, ainsi que le vide politique dans lequel ils se trouvent placés malgré eux, car on n'a tenu compte ni de leurs opinions ni de leurs sentiments.

La réalité politique nouvelle qui force les communautés francophones non québécoises à préciser leur identité les oblige également à réorienter leur action. À la suite du rejet du fédéralisme canadien par les États généraux du Canada français (1966-1967) et de la fondation du Mouvement Souveraineté-Association (1967), qui deviendra la principale composante du Parti québécois (1968), il n'est plus guère question de la survivance canadienne-française en Amérique; on parle plutôt de la souveraineté ou de l'indépendance d'un État du Québec qui transforme son Assemblée législative en Assemblée nationale (1968) et, au nom de ses intérêts collectifs, abandonne au Canada anglais les minorités qui ont été incorporées à ce dernier par la Constitution de 1867. L'abandon n'est pas complet, mais le changement d'attitude est si brutal et l'avenir appréhendé apparaît si noir que, tout en se plaignant du sort déplorable qui leur est fait, ces minorités se tournent immédiatement vers les seuls recours qui leur restent: leur volonté de vivre à tout prix, le regroupement de leurs ressources humaines, leurs gouvernements provinciaux et le gouvernement fédéral. 
La réaction de ces gouvernements est rapide et, jusqu'à un certain point, généreuse. En 1969, le Parlement fédéral vote la loi des langues officielles et le Secrétariat d'État, entre autres organismes fédéraux, prend des mesures pour aider financièrement la minorité anglophone du Québec et les minorités francophones des autres provinces. De 1969 à 1976, ce ministère accordera aux Franco-Ontariens $4418892 \$$ en subventions pour leurs centres culturels et pour l'animation sociale. Des gouvernements provinciaux font leur part. L'Ontario, par exemple, crée un bureau franco-ontarien à l'intérieur du Conseil des arts de la province (1969). Les organismes franco-ontariens avaient reçu 22500 \$ de ce conseil entre 1963 et 1970; pendant les six années suivantes, ils obtiendront $1088519 \$$, dont $40,9 \%$ pour les centres culturels et l'éducation artistique, $33 \%$ pour le théâtre, $7,8 \%$ pour la littérature et les publications ${ }^{16}$. Les Acadiens et les francophones de l'Ouest reçoivent aussi des sommes importantes des deux niveaux de gouvernement.

En même temps que ces ressources donnent un nouveau souffle à la vie culturelle, un néonationalisme naît; il sera plus revendicateur que l'ancien et il essaiera d'animer tous les secteurs et toutes les classes de la société. Les générations montantes et les classes populaires ne se contentent plus de ce qui a satisfait une élite que l'on juge bourgeoise et pas assez proche du peuple. Les institutions communautaires existantes doivent rajeunir leurs structures et se regrouper. L'Association canadienne-française d'éducation de l'Ontario (ACFÉO), fondée en 1910, se démocratise et se régio-

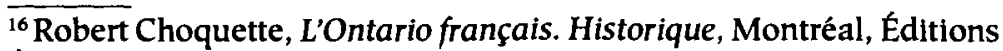
Etudes vivantes, «L'Ontario français», 1980, pp. 224-226. 
nalise en 1969 afin de mieux représenter l'ensemble de la collectivité; elle prend un nouveau nom: l'Association canadienne-française de l'Ontario (ACFO); vingt ans plus tard, elle regroupe vingt-deux sections régionales et dix-neuf associations diverses lui sont affiliées ${ }^{17}$. En 1973, la Société nationale des Acadiens, née en 1957 de la réorganisation de la Société nationale l'Assomption fondée en 1881, devient une fédération; elle comprend trois sociétés provinciales: la Société des Acadiens du Nouveau-Brunswick, la Fédération des Acadiens de la Nouvelle-Écosse, la Société Saint-Thomas-d'Aquin (Île-du-PrinceÉdouard) ${ }^{18}$. L'Association d'éducation des Canadiens français du Manitoba (AÉCFM) avait vu le jour en 1916, l'année où la loi Thornton, en faisant de l'anglais la langue officielle de l'enseignement, avait aboli le droit d'enseigner dans toute autre langue. En 1967, le français redevient langue d'enseignement (Loi 59) et, en 1970, il est mis, comme tel, sur le même pied que l'anglais (Loi 113). La situation changeant, l'AÉCFM cède sa place à la Société francomanitobaine $(1968)^{19}$, dont le champ de travail comprendra dorénavant tous les domaines de la vie communautaire, et à la Fédération provinciale des Comités de parents $(1976)^{20}$. Des changements se produisant

\footnotetext{
${ }^{17}$ Brigitte Bureau, Mêlez-vous de vos affaires. 20 Ans de luttes franco-ontariennes, [Vanier (Ontario)], l'Association canadiennefrançaise de l'Ontario, 1989, pp. 113-114.

${ }^{18}$ Marguerite Maillet, Histoire de la littérature acadienne. De rêve en rêve, Moncton, Éditions d'Acadie, «Universitaire», 1983, pp. 207, 213, 217.

${ }^{19}$ Annette Saint-Pierre, «Le Centre d'études franco-canadiennes de l'Ouest (CÉFCO). Collège universitaire de Saint-Boniface (Manitoba)", dans Quatre Siècles d'identité canadienne, actes d'un colloque tenu au Centre de recherche en civilisation canadienne-française de l'Université d'Ottawa, le 23 octobre 1981, présentés et publiés sous la direction de René Dionne, Montréal, Editions Bellarmin, 1983, pp. 151-152.

${ }^{20}$ Mon pays noir sur blanc. Regards sur le Manitoba français, textes et
} 
aussi dans la situation du français en Saskatchewan ${ }^{21}$ ainsi que dans les autres provinces et dans le Grand Nord, la population de langue française révise ses modes d'action et, avec l'aide du gouvernement fédéral, organise mieux la défense et la revendication de ses droits.

C'est ainsi que dans neuf provinces (le Québec s'étant exclu), au Yukon et dans les autres territoires du Nord, de très nombreuses associations de langue française, culturelles entre autres, naissent et établissent entre elles des liens indispensables, car il s'agit ni plus ni moins de réunir les forces vives de la minorité, quelles qu'elles soient et où qu'elles soient, en un faisceau dynamique qui luttera, non seulement pour une survie collective que l'on croit toujours possible, mais aussi pour une vie individuelle meilleure à l'intérieur d'un Canada anglais dont on veut jouir de tous les droits de citoyen. Il importe done au plus haut point de regrouper sur le plan national toutes ces associations et les francophones qu'elles représentent. C'est pourquoi, malgré l'existence du Conseil de la vie française en Amérique et d'associations pancanadiennes-françaises comme l'Association canadienne d'éducation de langue française qui continueront de compter des membres non québécois, la Fédération des francophones hors Québec, qui deviendra la Fédération des communautés francophones et acadienne du Canada en 1991, voit le jour en 1975; son rôle sera d'exprimer les besoins et les points de vue des diverses commu-

photos de Roger Turenne, Saint-Boniface (Manitoba), les Éditions du Blé, 1981, [s.p.]: "Une nouvelle question des écoles" (entre les photos numérotées 88 et 89 ).

${ }^{21}$ Jean-Guy Quenneville, «Les Fransaskois de la Saskatchewan», dans R. Dionne (dir.), Quatre Siecles d'identité canadienne, pp. 163-168. 
nautés françaises dont les intérêts diffèrent maintenant de ceux du Québec, qui avait été leur porte-parole jusque-là ${ }^{22}$.

Dans ce contexte neuf vont se faire entendre des voix littéraires nouvelles, engagées pour une bonne part, qu'appuieront soit des institutions déjà en place (organismes gouvernementaux ou communautaires, maisons d'enseignement, etc.), soit des institutions créées de toutes pièces (maisons d'édition, associations d'auteurs, etc.). Ainsi, peu à peu, se dégagent et émergent de la littérature canadienne-française de naguère trois littératures régionales, dont les corpus existaient depuis longtemps, mais ne jouissaient pas de leur indépendance, incorporés qu'ils étaient sous une appellation qui niait leurs identités particulières. Ce sont les littératures acadienne, franco-ontarienne et francophone de l'Ouest (celle-ci englobe les

\footnotetext{
${ }^{22}$ La Fédération "offre des programmes de coordination et de concertation, de fonctionnement, de recherche et d'information, de consultation et de développement. Ses principaux secteurs d'intervention touchent les communications, la constitution, l'économie, les loisirs et les sports ainsi que les services gouvernementaux. En outre, la Fédération publie régulièrement depuis sa création des dossiers sur différents aspects de la situation des francophones hors Québec." Charles Dufresne, Jacques Grimard, André Lapierre, Pierre Savard et Gaétan Vallières, Dictionnaire de l'Amérique française. Francophonie nord-américaine hors Québec, Ottawa, les Presses de l'Université d'Ottawa, 1988, p. 146. On trouvera dans ce dictionnaire des renseignements sur la plupart des associations, fédérations et sociétés que nous avons citées plus haut ou citerons ci-après. - Pour connaître la situation du français en Alberta et dans le Grand Nord à la même époque, voir les chapitres signés par Maurice Lavallée (pp. 37-42: "L'Enseignement bilingue en Alberta depuis les années 1920») et Marie Moser (pp. 77-98: «Le Groupe canadien-français d'Edmonton et des environs. Ses caractéristiques selon l'Ouest canadien (1898-1900)"), dans Aspects du passé franco-albertain. Témoignages et études, sous la direction de Alice Trottier, Kenneth J. Munro et Gratien Allaire, Edmonton, le Salon d'histoire de la francophonie albertaine, "Histoire franco-albertaine", 3, 1980, iv[3], $146 \mathrm{p}$.
} 
littératures franco-manitobaine, fransaskoise, franco-albertaine, franco-colombienne, francoyukonaise, franco-ténoise). Ces qualificatifs ne sont pas nouveaux, mais leur dénotation devient plus spécifique lorsqu'ils servent à qualifier soit des identités que le terme québécois ne peut plus absorber, soit des littératures qui s'affranchissent de la québécoise et s'enracinent dans des terres régionales sans les soustraire pour autant à leur canadianité. Ainsi affranchies, ces trois littératures vont connaître un élan nouveau sous le triple signe de la contestation, de la récupération et de l'institutionnalisation.

\section{II - LA CONTESTATION}

Les Acadiens se sont toujours considérés comme différents des Québécois. Depuis le milieu du dix-neuvième siècle, ils se sont efforcés, suivant en cela l'exemple du Québec, de produire une littérature qui les identifierait comme nation distincte en témoignant de leurs origines et de leur histoire particulière. Les œuvres produites avant 1958 reconstruisent donc l'Acadie depuis ses débuts de terre promise sous le régime français. Fondées principalement sur la tragique déportation de $\mathbf{1 7 5 5}$ et les difficultés d'un retour courageux, ces œuvres relativement nombreuses constituent un patrimoine précieux qui sert l'idéologie de la survivance avant tout. Au cours de la décennie de 1960, une Acadie nouvelle se lève: jeune, elle regarde l'avenir. Celui-ci paraît s'ouvrir au Nouveau-Brunswick avec l'élection d'un premier ministre acadien qui promet "des chances égales pour tous» (1960), permet la fondation de l'Université de Moncton (1963), rend possible la création d'une école normale française (1968) et fait recon- 
naître le français comme l'une des deux langues officielles de la province (1969).

Dès 1968, selon Marguerite Maillet, historienne de la littérature acadienne, l'Université de Moncton devient un lieu de contestation. Des jeunes en quête d'identité, "sensibilisés aux problèmes des minorités" et "aux maux engendrés par le capitalisme et la technologie», "lanc(ent) les débats nationaux sur la place publique [...] chantent leur révolte, et leur libération anticipée, dans des nuits de poésie inoubliables $^{23}$ ". Ils ne veulent pas d'un pays qui ne serait que mythique et ils rejettent l'image de l'Acadien "passif et soumis ${ }^{24} »$. Ils font appel au réalisme, démasquent les oppresseurs, flagellent les peureux et les silencieux; ils dénoncent l'anglicisation que l'on est porté à accepter pour un petit pain, prophétisent un avenir catastrophique, qu'ils décrivent au présent ${ }^{25}$. Il n'en reste pas moins que cette poésie exprime «en même temps que la souffrance et l'humiliation d'un peuple un immense amour pour un pays à faire naître à la vraie liberté ${ }^{26} \%$.

En Ontario français, ce sont des jeunes du nord de la province qui ouvrent le bal de la contestation. Ils ont étudié les humanités au Collège du Sacré-Cœur de Sudbury ou au Collège universitaire de Hearst, les lettres à l'université Laurentienne (bilingue) fondée

\footnotetext{
${ }^{23}$ M. Maillet, Histoire [...], p. 179.

${ }^{24}$ Ibid., p. 188.

${ }^{25}$ La dénonciation se fait particulièrement violente chez Guy Arsenault (voir "People in a room", Acadie Rock, Moncton, les Éditions d'Acadie, 1973, pp. 68-71) et Herménégilde Chiasson (voir "Jaune», Mourir d Scoudouc, Moncton, les Éditions d'Acadie, 1974, p. 44). Voir aussi M. Maillet, "La Poésie acadienne contemporaine et la contestation", Revue d'histoire littéraire du Québec et du Canada français [désormais: RHLQCF], $\mathrm{n}^{\circ}$ 3, hiver-printemps 1982, pp. 112-123.
}

${ }^{26}$ M. Maillet, Histoire [...], p. 193. 
en 1960. Ne sachant pas qu'il existe des œuvres franco-ontariennes, car leurs professeurs n'ont jamais parlé que de littérature française ou de littérature canadienne-française, ils se sentent pauvres depuis que les Québécois ont confisqué cette dernière, qu'ils croyaient être aussi la leur, sous une appellation exclusive. En effet, comme les jeunes Québécois des années 1830 qui s'imaginaient que «la littérature fonde la gloire des peuples ${ }^{27}$ ), ils pensent, formés eux aussi dans les lettres classiques, que la littérature donne une identité à ceux qui n'en ont pas, faute de l'avoir exprimée. Ils se tournent donc vers l'écriture poétique.

À deux ou trois exceptions près ${ }^{28}$, leurs œuvres ne contiennent pas la violence des poètes acadiens. Une bonne partie n'en dénoncent pas moins une situation morne et pénible. Leurs auteurs étalent parfois à plat $^{29}$, parfois de façon plus colorée ${ }^{30}$, l'état d'infériorité et de pauvreté dans lequel vit la communauté franco-ontarienne. C'est la propagande du pire, qui ne peut qu'inciter à la révolte les âmes bien nées; aussi seront-elles légion à refuser l'image que cette poésie véhicule pour leur plus grande honte. Même si la plupart exprimèrent ce refus en s'engageant dans

\footnotetext{
${ }^{27}$ [Hyacinthe-Poirier Leblanc de Marconnay], «À la jeunesse canadienne» [Prospectus], le Populaire, 10 avril 1837. Le texte est reproduit en entier dans R. Dionne, La Patrie littéraire, 1760-1895, volume II de l'Anthologie de la littérature québécoise, sous la direction de G. Marcotte, Montréal, La Presse, 1978, pp. 28-30; [2 éd. révisée], Montréal, L'Hexagone, t. I, vol. 2, pp. 328-330.

${ }^{28}$ Exemple: Réginald Bélair, Éclipses, [Kapuskasing, Éditions du Centre régional de loisirs culturels, 1973?], 24 p.

${ }^{29}$ Exemples: Patrice Desbiens, l'Espace qui reste, Sudbury, Prise de parole, 1977, 47 p.; Sudbury. Textes 1981-1983, Sudbury, Prise de parole, «Poésie», 1983, 63 p.

${ }^{30}$ Exemple: Jean-Marc Dalpé, Gens d'ici, avec des dessins de François-X. Chamberland, Sudbury, Prise de parole, «Poésie», 1981, 94[2] p.
} 
une action positive avec une ferveur renouvelée, il reste que, en quelques occasions, l'opinion publique franco-ontarienne réclama la censure de poèmes jugés mal représentatifs ou écrits dans une langue bâtarde dite populaire, parce que misérabilistes diront des aînés qui soignent leur réputation, en réalité parce que d'une vérité trop accusatrice répliqueront les jeunes militants socialistes ou néonationalistes ${ }^{31}$. Un bon nombre de poètes de l'époque écrivent plutôt pour mieux vivre leur solitude: ils essaient de se fondre harmonieusement dans la grande nature du Nord $^{32}$, confient leurs amours difficiles ou chantent l'amitié ${ }^{33}$. Pour eux aussi, le dire poétique est affirmation d'identité, car il exprime l'isolement dans lequel survit une collectivité en mal d'amour et d'estime autant que d'égalité.

À la fin des années 1960, les francophones de l'Ouest s'éveillaient de plus en plus à l'existence d'une littérature canadienne-française. L'abondance et la qualité des œuvres publiées sous cette appellation depuis le Bonheur d'occasion (1945) de Gabrielle Roy leur permettaient d'affirmer leur identité et de spécifier leur appartenance séculaire dans un Canada anglais qui

${ }^{31}$ Ce fut le cas lors de la publication à l'occasion de Noël, dans un journal de l'ACFO, le Temps, du poème "Gerry Brault» de J.-M. Dalpé, Gens d'ici, pp. 75-81. Autre cas semblable: beaucoup d'habitants de Hawkesbury n'acceptèrent pas facilement la chanson «la Toune d'Hawkesbury" qui servait d'ouverture et de finale à la pièce de Brigitte Hæentjens et J.-M. Dalpé, Hawkesbury Blues, Sudbury, Prise de parole, «Théâtre», 1982, pp. 6, 73-74. Le texte est reproduit dans R. Dionne, Anthologie de la poésie franco-ontarienne, des origines d nos jours, Sudbury, Prise de parole, 1991, pp. 156-157.

${ }^{32}$ Exemples: Guy Lizotte, Cicatrice, [illustrations de Laurent Vaillancourt], Sudbury, les Éditions Prise de parole, 1977, 71 p. ; La Dame blanche, [illustrations de Laurent Vaillancourt], Hearst, les Éditions Boréales, $1981,59 \mathrm{p}$.

${ }^{33}$ Exemple: Michel Vallières, Comme un simple voyageur, Sudbury, Prise de parole, "Poésie», 1984, 46[1] p. 
prenait lui aussi conscience de son identité particulière à travers ses propres œuvres qu'il commençait enfin d'enseigner. Leur fierté fut profondément blessée lorsque survint la séparation littéraire du Québec. Historienne de la littérature francophone de l'Ouest, Annette Saint-Pierre a bien exprimé le choc qui secoua ses compatriotes et le désarroi qui s'ensuivit:

Périodiques, journaux, livres, émissions télévisées et radiodiffusées devenaient les véhicules d'une littérature "québécoise» qui s'affichait avec vigueur et confiance. La nouvelle appellation s'expliquait ainsi: ce qui s'écrivait en français au Canada s'écrivait surtout au Québec, où l'on vivait des heures cruciales pour la création d'une identité à laquelle aspirait une bonne partie de la population.

Pour celui qui voyait la littérature canadienne comme un immense creuset, alimenté de temps à autre par l'apport appréciable des écrivains d'au-delà des frontières, le choc fut dur. En effet, la nouvelle étiquette, «littérature québécoise», allait signifier au Canadien «hors Québec» qu'il vivait dans un milieu "sans littérature». Cette soudaine amputation culturelle allait provoquer une réflexion bénéfique sur la contribution de l'Ouest aux lettres canadiennes.

Un peuple sans littérature est-il encore un peuple? Toute société n'a-t-elle pas une âme, une conscience, un cœur ou une vie de l'esprit qui s'exprime par la voix de l'écriture? L'Ouest canadien serait-il un peuple sans cour et sans âme? Quels sont les rêves, les aspirations, les joies, les peines des Franco-Colombiens, des Franco-Albertains, des Fransaskois et des Franco-Manitobains? Quelles sont leurs difficultés de vivre et de durer «hors Québec"? Comment découvrir la véritable identité des francophones de l'Ouest? ? $^{34}$

Certains refusent de répondre à la question: ils s'exilent en terres étrangères ou se réfugient soit dans une autre langue, soit dans une écriture qui serait

${ }^{34}$ «L'Ouest canadien et sa littérature», $R H L Q C F, \mathrm{n}^{\circ} 12$, été-automne 1986, pp. 171-172. On aura remarqué que, dans le texte cité, le terme «canadien» équivaut à «canadien-français». 
son propre et unique lieu de référence et ainsi permettrait d'«entrer, par l(a) spécificité d'expression, dans la généralité de l'écriture du monde ${ }^{35}$ ». Quant aux autres, les plus nombreux, il s'écoulera quelque cinq années avant qu'ils réagissent en force à la situation nouvelle, et quand ils le feront, ce sera tout simplement pour écrire en toute modestie et sans récrimination, à la façon de leurs devanciers de la première moitié du siècle. Ils ne contesteront rien ni personne, mais ils continueront la lutte pour la vie avec une volonté décuplée de dire qui ils sont et ce qu'ils vivent $^{36}$, et ils le feront en sachant qu'ils ne peuvent plus guère compter que sur eux-mêmes ainsi que sur la sympathie et l'aide des minorités francophones de l'Ontario et de l'Acadie qui se sont mises à créer intensément depuis 1972.

\section{III - LA RECUPÉRATION}

Si la contestation a donné une orientation nouvelle à la littérature de ces deux dernières régions, elle n'a pas empêché que s'y continue, tout comme dans l'Ouest, une littérature d'illustration et de vénération d'un passé commun, plus ou moins lointain, que les contestataires devaient forcément rappeler et décrire, eux aussi, mais avec une attitude négative, pour mieux en amener le rejet. Il se trouva des lecteurs pour les deux courants littéraires, et des chercheurs (critiques, professeurs, historiens) comprirent assez tôt qu'il n'y a pas de présent qui subsiste sans passé, même quand s'ouvre un avenir différent. Une littérature ne saurait exister, en effet, sans une tradition

${ }^{35}$ J[oseph] R[oger] Léveillé, Anthologie de la poésie franco-manitobaine, Saint-Boniface (Manitoba), les Éditions du Blé, 1990, p. 72.

${ }^{36}$ A. Saint-Pierre, «L'Ouest canadien et sa littérature", pp. 190-198. 
d'écriture et de lecture. On discerna donc bientôt un mouvement de récupération semblable à ceux que les littératures québécoise et acadienne avaient connu, avec un bonheur inégal, dans une première tentative de fondation durant la deuxième moitié du dix-neuvième siècle.

Comme en ce temps-là, c'est vers le folklore et la petite histoire que l'on se tourne d'instinct pour se constituer un patrimoine et donner de la profondeur à ses racines. Des folkloristes de carrière, tels Anselme Chiasson ${ }^{37}$ en Acadie et Germain Lemieux ${ }^{38}$ en Ontario, ou des musiciens comme Marcien Ferland ${ }^{39}$ au Manitoba, publient les résultats de leurs enquêtes: des légendes, des contes, des chansons. D'autres, en même temps, s'emploient à faire connaître et revivre les traditions populaires; ils pratiquent le récit folklorique comme Régis Brun $^{40}$ ou historique comme Louis Haché ${ }^{41}$ et Marius Benoist ${ }^{42}$. On trouve et publie de vieux manuscrits qui mettent des lecteurs avides de leurs racines en présence d'un passé vivant ${ }^{43}$, on prête sa plume à un vieux conteur qui narre sa vie ${ }^{44}$

${ }^{37}$ Les Légendes des Îles de la Madeleine, Moncton, Éditions des Aboiteaux, 1969, 126 p.; Le Diable Frigolet et 24 autres contes des îles de la Madeleine, Moncton, Éditions d'Acadie, [1991], 224 p.; etc.

${ }^{38}$ Les vieux m'ont conté, Montréal, les Éditions Bellarmin, et Paris, Maisonneuve et Larose, 1973-1991, 33 vol.; etc.

${ }^{39}$ Chansons à répondre du Manitoba, recueillies et présentées par Marcien Ferland, Saint-Boniface (Manitoba), les Éditions du Blé, 1979, xviii, 218 p.

${ }^{40} \mathrm{La}$ Mariecomo, roman, Montréal, Éditions du Jour, «Les Romanciers du Journ, R-112, 1974, 129 p.; etc.

${ }^{41}$ Charmante Miscou, Moncton, les Éditions d'Acadie, 1974, 115 p.; etc. ${ }^{42}$ Louison Sansregret, métis, un récit historique, illustrations de Suzanne Gauthier, Saint-Boniface (Manitoba), les Éditions du Blé, 1975, [viii], 94 p. ${ }^{43}$ Gilles Martel, Glen Campbell et Thomas Flanagan, Louis Riel. Poésies de jeunesse, Saint-Boniface (Manitoba), les Éditions du Blé, 1977, x, 160 p. ${ }^{44}$ Guillaume Charette, L'Espace de Louis Goulet, Winnipeg (Manitoba), Éditions Bois-Brûlés, 1976, 201 p. 
et on adapte ou réécrit en français moderne des légendes et des contes anciens ${ }^{45}$.

Des créateurs fondent leurs œuvres de fiction sur l'histoire de leur région et les mœurs et coutumes de ses habitants. Les Acadiens d'hier et de naguère revivent dans les pièces réalistes ${ }^{46}$ et les romans à la fois folkloriques et mythiques d'Antonine Maillet ${ }^{47}$. Hélène Brodeur rédige le premier tome de ses «chroniques du Nouvel Ontario ${ }^{48}$ ", fresque romanesque qui repose sur l'histoire rigoureusement documentée du développement de cette région de 1913 à 1968 . Gabrielle Roy publie sous forme de nouvelles des portraits souvenirs que l'utilisation d'une seule narratrice pour l'ensemble des textes invite à lire comme un roman, Ces enfants de ma vie ${ }^{49}$, "un autre présent de l'auteure à ses compatriotes franco-manitobains ${ }^{50}$ ".

Très tôt, il se trouva des universitaires pour s'intéresser aux nouvelles littératures régionales. Comme

${ }^{45}$ Melvin Gallant, Ti-Jean, contes acadiens, Moncton, les Editions d'Acadie, 1973, 165[1] p.; Yvonne Lagassé (en collaboration avec Roger Lagassé), Saint-Boniface, Manitoba, Editions des Plaines, 1981, 55 p.; id., Les Trois Pommes d'or, Saint-Boniface, Manitoba, Editions des Plaines, $1982,40 \mathrm{p}$.

${ }^{46}$ Les Crasseux, [présentation de Jacques Ferron], Montréal, Holt, Rinehart et Wilson, «Théâtre vivant», $5,1968,68$ [1] p.; [2 $2^{\mathrm{e}}$ éd.], pièce en trois actes, présentations de Rita Scalabrini et Jacques Ferron, Montréal, Leméac, «Répertoire acadien», 1, 1973, xxxiii, 91[6] p.; la Sagouine, pièce pour une femme seule, Montréal, Leméac, "Répertoire acadien», 1/2, 1971, 105[1] p.; etc.

${ }^{47}$ Don l'Orignal, roman, Montréal, Leméac, "Roman acadien», 1, 1972, 149 p.; Pélagie-la-Charrette, roman, Montréal, Leméac, «Roman», 30, 1979,351 p.; etc.

${ }^{48}$ La Quête d'Alexandre, Montréal, Quinze, "Prose entière», 283 p.; [ [2 éd.], Sudbury, Prise de parole, 1985, 283[2] p.; Entre l'aube et le jour, Montréal, Quinze, "prose entière», 1983, 200 p.; [2 éd.], Sudbury, Prise de parole, 1986, 233 p.; Les Routes incertaines, Sudbury, Prise de parole, 1986, 233[2] p.

${ }^{49}$ Ces enfants de ma vie, Montréal, Stanké, 1977, 212 [1] p.

${ }^{50} \mathrm{~A}$. Saint-Pierre, "L'Ouest canadien et sa littérature», p. 188. 
plusieurs d'entre eux avaient une formation en histoire littéraire, c'est tout naturellement qu'ils entreprirent de récupérer à leur façon le patrimoine des créateurs. Assez rapidement, pour répondre aux besoins immédiats des enseignants, ils compilèrent des dictionnaires ${ }^{51}$ ou des répertoires d'auteurs ${ }^{52}$, des bibliographies partielles ${ }^{53}$ ou préliminaires ${ }^{54}$, des recueils de textes choisis (à l'usage des écoles) ${ }^{55}$, des anthologies ${ }^{56}$, etc. Avec des vues plus lointaines, certains commencèrent des recherches qui devaient aboutir à la reconnaissance de corpus littéraires dont on ne pourrait contester ni les fondements séculaires ni la fécondité. Les résultats ne seraient connus que partiellement durant la décennie de 1970 , mais les années 80 verraient l'achèvement ou la parution d'outils de travail ${ }^{57}$ et d'études

${ }^{51}$ M. Gallant et Ginette Gould, Portraits d'écrivains. Dictionnaire des écrivains acadiens, Moncton, les Éditions d'Acadie, 1980, $182 \mathrm{p}$.

${ }^{52} \mathrm{G}$ [isèle] D[upasquier] et S[imone] M[archildon], Auteurs francophones des Prairies, Saint-Boniface (Manitoba), Centre de ressources éducatives françaises du Manitoba, 1981, iii, 47 p.

${ }^{53}$ Alexandre L. Amprimoz, "Bibliographie de la poésie francocanadienne de l'Ouest», Bulletin du Centre d'études franco-canadiennes de l'Ouest, $\mathrm{n}^{\circ}$ 2, mai 1979, pp. 9-14; M. Maillet, «Littérature acadienne (1874-1960)", [R]HLQJCF], n' 1, 1979, pp. 92-98; etc.

${ }^{54} \mathrm{R}$. Dionne, Bibliographie de la littérature outaouaise et franco-ontarienne, édition préliminaire, Ottawa, Université d'Ottawa (CRCCF), «Documents de travail du Centre de recherche en civilisation canadienne-française", 10, 1978, 91 p.; $2^{\mathrm{e}}$ éd. révisée et augmentée, 1981 , viii, $204 \mathrm{p}$.

${ }^{55}$ Voir la liste franco-ontarienne dans $\mathrm{R}$. Dionne, La Littérature régionale aux confins de l'histoire et de la géographie, Sudbury, Prise de parole, «Ancrages», 1993, p. 77, note 85.

${ }^{56}$ Voir ibid., les listes acadienne (p. 65, note 34 ) et franco-ontarienne (p. 77, note 87).

${ }^{57}$ Le Centre d'études acadiennes [de l']Université de Moncton, Inventaire général des sources documentaires sur les Acadiens, 3 vol., Moncton, Éditions d'Acadie, 1975, 1976 et 1977, 526, xili, 463 et vii, 212 p.; Guide bibliographique de l'Acadie, 1976-1987, œuvre réalisée sous la direction de Muriel Kent Roy, Moncton, Centre d'études acadiennes, 
importantes $^{58}$. En cours de route, des miettes tomberaient de la table de ces chercheurs; on les retrouve sous forme de chapitres de livres, d'articles de revues, de communications données lors de colloques savants ou, tout simplement, de conférences et de causeries. Des publics divers allaient se former, qui utiliseraient les outils de travail ou liraient les œuvres.

\section{IV - Des institutions NOUVELLES OU RENOUVELÉES}

Animés par une ambition commune: la promotion de l'identité spécifique de communautés dont ils voulaient améliorer la situation socioculturelle et politique, contestataires et récupérateurs devaient s'adresser en chœur à tous les secteurs de la population. Aussi s'employèrent-ils, d'une part, à créer des institutions et, d'autre part, à occuper une place meilleure dans celles qui existaient déjà, car, il ne faut pas l'oublier, depuis des décennies ou plus d'un siècle un processus d'institutionnalisation était en marche: des œuvres avaient été créées, des périodiques existaient, des bribes d'enseignement se donnaient, des associations littéraires ou culturelles étaient en place, etc. Et ces institutions avaient montré leur efficacité en rassemblant les forces collectives

1988, xvii, $508 \mathrm{p}$. [ce volume et les trois précédents contiennent des sections importantes consacrées spécifiquement à la littérature]; Propos sur la littérature outaouaise et franco-ontarienne, introduction et choix de textes par R. Dionne, vol. 1, Ottawa, Université d'Ottawa (CRCCF), "Documents de travail du Centre de recherche en civilisation canadienne-française», 11, 1978, 209 p.; vol. 2, Ottawa, la Société des écrivains canadiens (section d'Ottawa-Hull), 1979, 215 p.; vol. 3, Ottawa, Université d'Ottawa (CRCCF), 1981, 237 p.; vol. 4, 1983, 324 p.; etc.

${ }^{58}$ Voir R. Dionne, La Littérature régionale [...], pp. 64-67 (Acadie), pp. 7679 (Ontario français) et pp. 79-82 (Ouest canadien). 
sous le drapeau de la survivance par la fidélité à la langue, a la religion catholique et aux traditions importées de France ou créées depuis l'établissement de la Nouvelle-France. Le journal l'Evangéline (1887-1981), par exemple, comme l'a bien fait voir James de Finney, «en plus de jouer le rôle de journal national des Acadiens, (a participé) activement à la vie littéraire» au point qu'il «fait figure de laboratoire et de microcosme de la vie littéraire en Acadie $^{59}$ ». En utilisant une méthode semblable à celle du professeur de Moncton, on pourrait facilement montrer que le Droit (fondé à Ottawa en 1913), la Liberté (fondée à Winnipeg en 1913) et la Survivance (fondée à Edmonton en 1928), entre autres journaux, ont joué un rôle important dans la vie littéraire des francophones de l'Ontario et de l'Ouest canadien. Plusieurs journaux (ou leurs imprimeries) se sont même transformés occasionnellement en éditeurs durant les périodes où ceux-ci se faisaient rares dans leur région. La situation change au début des années 1970.

En 1972, des professeurs de l'Université de Moncton fondent les Éditions d'Acadie. En 1979, celles-ci ont déjà publié 16 ouvrages de 13 auteurs nouveaux ${ }^{60}$; huit ans plus tard, leur catalogue contiendra une centaine de titres, et en 1992 plus de deux cents. En 1973, les jeunes auteurs du Nord ontarien, aidés par des professeurs de l'université Laurentienne et de l'Université de Sudbury, lancent le premier livre des

\footnotetext{
${ }^{59}$ «Le Journal L'Évangéline et l'émergence de l'institution littéraire acadienne», Francophonies d'Amérique, n 1, 1991, p. 43.

${ }^{60} \mathrm{M}$. Maillet, "L'Acadie, une mémoire", dans Literatures of Lesser Diffusion/Les Littératures de moindre diffusion, edited by Joseph Pivato, in collaboration with Steven Tötösy de Zepetnek and Milan V. Dimic, with the assistance of Charlotte Garrett and Lise-Anne Lavigne, proceedings of a conference, Edmonton, University of Alberta, Research Institute for Comparative Literature, 1990, p. 107.
} 
Éditions Prise de parole; en 1982, leur fonds comprendra une quarantaine d'ouvrages, le double en 1989, et une centaine trois ans plus tard. À Saint-Boniface, les Éditions du Blé naissent en 1974 à l'instigation, entre autres, de professeurs du Collège universitaire; quatorze ans plus tard, elles auront publié une soixantaine de volumes, et le rythme de production se maintient. En 1979, deux membres du comité d'administration quittent la maison pour créer les Éditions des Plaines, dont le fonds compte une centaine de volumes en 1992. Dans chaque région, des maisons plus petites naîtront au cours de la décennie de $1980^{61}$. La plupart existent encore aujourd'hui et leur contribution est parfois considérable.

Conséquence de la fondation de ces maisons, les journaux publieront de moins en moins de textes de création, mais ils consacreront plus d'espace à la présentation et à la critique des ouvres durant la décennie de 1970; au cours de la décennie suivante, la part de la critique diminue. Les professeurs qui en étaient souvent les auteurs ont maintenant des débouchés qui les intéressent davantage: les revues savantes $^{62}$ et les bulletins ${ }^{63}$, qu'ils ont fondés sur une

${ }^{61}$ En Acadie: les Éditions Perce-Neige [Moncton (N. B.), 1980], les Editions Lescarbot [Yarmouth (N. E.), 1980], etc.; en Ontario: les Éditions L'Interligne [Ottawa, 1981], les Éditions du Vermillon [Ottawa, 1982], les Éditions du GREF [Toronto, 1984], les Éditions Le Nordir [Hearst, 1988], etc.; dans l'Ouest: les Éditions Louis-Riel [Regina (Saskatchewan), 1984], etc. Voir une liste plus complète, mais non exhaustive, dans $\mathrm{R}$. Dionne, La Littérature régionale [...], pp. 85-87. ${ }^{62}$ La Revue de l'Université de Moncton (1968), Si que (Université de Moncton, 1976-1979, 1983-1984), Revue de l'Université Sainte-Anne (1977), Revue du Nouvel-Ontario (Université Laurentienne, 1978), Cahiers franco-canadiens de l'Ouest (Collège universitaire de Saint-Boniface, 1989), etc.

${ }^{63}$ Bulletin du Centre de recherche en civilisation canadienne-française de 
base régionale et qui consacrent à la littérature soit des numéros, soit des chroniques régulières, et les magazines culturels qui sont nés pour servir à la fois les éditeurs et le grand public ${ }^{64}$. Des revues de création littéraire se créent, de poésie surtout. Éphémères ${ }^{65}$ à quelques exceptions près ${ }^{66}$, elles concourent quand même, le temps qu'elles durent, à la production littéraire. La grande majorité des journaux ne publiant plus de poésie, elles servent souvent de banc d'essai à des poètes, jeunes la plupart du temps, qui, bien reçus par la critique, oseront lancer par la suite un premier recueil ${ }^{67}$.

L'enseignement des trois littératures sous leur nom spécifique commencera tardivement. Avant la fin des années 1960, les textes de la littérature française occupent presque toute la place dans les programmes de lettres et de langue des institutions d'enseignement. À tous les niveaux (primaire, secondaire, collégial et universitaire), il se trouve cependant que des enseignants et des professeurs utilisent depuis longtemps, bien qu'en nombre restreint, des textes canadiens-français. Une grande partie des conseils de direction et des corps professoraux s'opposent quand même à une utilisation plus importante de ces textes, arguant que les œuvres canadiennes n'ont pas la valeur des œuvres françaises: le contenu est pauvre, la langue est déficiente ${ }^{68}$, etc. C'est pourquoi,

l'Université d'Ottawa (1970-1983), Bulletin du Centre d'études francocanadiennes de l'Ouest (1979-1988), etc.

${ }^{64}$ Liaison (Ottawa, 1978), etc.

${ }^{65}$ Prosepectus (Ottawa, 1978-1979), Rauque, (Sudbury, 1984-1987), etc.

${ }^{66}$ Éloizes (Moncton, 1980), Callicriture (Moncton, 1989), etc.

${ }^{67}$ Alain-Bernard Marchand, Claire Rochon, Judith Hamel...

${ }^{68}$ La promotion de la littérature régionale n'était certes pas aidée par le fait que, à cette époque, certains lettrés, des écrivains entre autres, 
même si l'on utilise, depuis la décennie de 1950, des méthodes pédagogiques nouvelles, concrètes, qui font d'abord découvrir à l'élève la géographie de son milieu environnant avant celui de l'Europe, on refuse de lui enseigner, non seulement d'abord, mais aussi par la suite, autre chose que des fragments de sa littérature, et encore est-ce en lui signifiant qu'elle n'a guère de valeur que folklorique et sentimentale. Il apparut alors assez clairement que les littératures régionales ne pourraient être reconnues pleinement que si on les enseignait au niveau universitaire.

Les Acadiens, maîtres dans une université à eux, furent les premiers à inscrire des cours de littérature régionale dans leur programme d'études françaises. En 1973, on pouvait suivre à l'Université de Moncton un cours de littérature acadienne et un cours de folklore acadien; en 1988, le même programme comprenait «quatre cours de littérature écrite et deux de folklore au niveau du baccalauréat» ainsi qu'un séminaire de maîtrise et "six thèses en littérature acadienne avaient été soutenues [...] depuis $1975^{69} »$. Le secteur «Littérature» du programme de doctorat en études françaises, établi à l'automne de 1989, comprend cinq cours de littérature tandis que, dans six des sept cours du secteur «Linguistique», une grande partie de la matière est acadienne, même si un seul intitulé de cours inclut ce qualificatif ${ }^{70}$. L'Université

encourageaient l'utilisation du «joual» québécois ou du parler populaire dans les œuvres de fiction.

${ }^{69}$ M. Maillet, "L'Acadie, une mémoire", p. 108. Avant 1979, au moins dix thèses en littérature acadienne avaient été soutenues dans d'autres universités à l'intérieur de programmes intitulés autrement (voir $\mathrm{M}$. Maillet, Histoire $[. .$.$] , p. 250).$

${ }^{70}$ D'après le texte [inédit] d'une conférence prononcée par Marguerite Maillet à l'Université de Moncton le 23 mars 1990; intitulée "Doctorat en études françaises à l'Université de Moncton. Couronnement et 
Sainte-Anne offre deux cours et deux séminaires de littérature acadienne écrite ainsi qu'un cours de littérature orale.

En Ontario, où les Franco-Ontariens ne possèdent pas encore leur université française, la situation est loin d'être aussi bonne, même si la clientèle est beaucoup plus abondante qu'en Acadie. Au point de départ, il y eut le zèle de quelques professeurs, puis la fondation, en 1976, du Groupe interuniversitaire d'études franco-ontariennes, composé de professeurs de trois universités et de deux collèges universitaires. Le Groupe obtint du ministère des Collèges et Universités des sommes qui permirent de créer des cours et d'organiser des colloques sur les réalités franco-ontariennes ${ }^{71}$. Ce n'est qu'en 1975-1976, que furent établis au département des lettres françaises de l'Université d'Ottawa les premiers cours de littérature franco-ontarienne: l'un au niveau de la maîtrise et du doctorat et l'autre au niveau du baccalauréat ; à ce dernier niveau, deux autres cours s'ajouteront en 1991. Il avait fallu attendre 1977 pour qu'un premier cours soit offert ${ }^{72}$. Le programme comprend aussi un cours intitulé «Autres Expressions littéraires en Amérique du Nord»; offert pour la première fois en 1985, il a porté à deux reprises sur la littérature de l'Ouest canadien et une fois sur la littérature louisianaise. Au département de français de l'université Laurentienne, on installa, en 1976, quatre

tremplin" (tapuscrit, 16 p.), cette conférence contient des renseignements historiques abondants et précis sur l'enseignement de la littérature acadienne, les circonstances qui en ont favorisé le développement et les projets de recherches en cours.

${ }^{7}$ R. Dionne, «G.I.E.F.O. (Groupe interuniversitaire d'études franco-ontariennes)", Bulletin du Centre de recherche en civilisation canadienne-française de l'Université d'Ottawa, no 18 , avril 1979, pp. 1-4. ${ }^{72}$ Id., «Le G.I.E.F.O. à l'Université d'Ottawa», ibid., pp. 5-6. 
cours "de linguistique portant sur la langue franco-ontarienne ${ }^{73} "$, mais aucun cours de littérature proprement dite, sauf si l'on considère comme tel celui intitulé "Essai de création littéraire», qui disparut par la suite. En 1991, le programme de littérature du département ne comprenait aucun cours de littérature franco-ontarienne, même si certains professeurs utilisaient des œuvres francoontariennes, entre autres dans des cours dont l'intitulé incluait le qualificatif "canadien-français». En 1992, le département de français a créé cinq cours de littérature franco-ontarienne écrite et le département de folklore de l'Université de Sudbury, où un programme de folklore existe depuis 1975, offre neuf cours de littérature orale. Au Collège universitaire Glendon, on enseigne la littérature franco-ontarienne et la littérature acadienne dans deux cours qui ont pour titre «les Minorités hors Québec»; on avait commencé à parler de ces littératures dès 1975-1976 dans un cours de civilisation canadienne-française. Depuis 1988, il existe un cours de littérature franco-ontarienne à l'Université de Guelph.

Pendant la décennie de 1960, Roger Motut, professeur à l'Université de l'Alberta, aurait été le premier à enseigner des œuvres franco-canadiennes de l'Ouest, mais c'est à l'intérieur d'un cours de littérature canadienne qu'il doit le faire; il s'emploie à faire connaître les auteurs, dirige des thèses de maîtrise sur leurs œuvres et rédige sa thèse de doctorat sur un écrivain francophone de l'Ouest. En 1992, le programme de l'Université de l'Alberta comprend deux cours de littérature régionale: «French Canadien Literature outside Quebec" et "Littérature

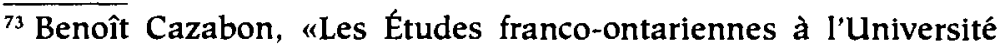
Laurentienne. Trois Années de travail», ibid, pp. 7-9. 
canadienne-française hors du Québec». Quant au Collège universitaire de Saint-Boniface, où l'on enseignait, dès 1968, des auteurs de l'Ouest dans les cours de littérature canadienne (cinq ont été créés entre 1968 et 1976), il offre, depuis 1980 , un cours intitulé «Littérature de l'Ouest».

Dans chacune des trois régions, des colloques tenus spécifiquement sur la littérature régionale et son contexte culturel ont contribué à fonder cet enseignement. Les Centres de recherches et d'études en civilisation canadienne-française, surtout depuis la formation de leur Regroupement en $1981^{74}$, ont servi de points d'appui à la plupart de ces colloques. Au moins cinq, dont l'un a attiré 65 conférenciers et 1000 participants $^{75}$ ont eu lieu en Acadie, six en Ontario ${ }^{76}$, onze dans l'Ouest ${ }^{77}$, et les actes de presque tous ont été publiés en tout ou en partie dans des livres ou des revues.

\footnotetext{
${ }^{74}$ Voir R. Dionne (dir.), Quatre Siècles d'identité canadienne, 176 p.

${ }^{75}$ Les actes de ce colloque, tenu à l'Université de Moncton du 11 au 13 mai 1978, ont été publiés en grande partie dans la Revue de l'Université de Moncton, vol. 11, n $\mathrm{n}^{\circ}$, mai 1978, 128 p., et dans les Cahiers de la Société historique acadienne. Quatre autres colloques ont également donné lieu à une publication: Neil-J. Boucher (dir.), les Régions acadiennes de la Nouvelle-Écosse. Histoire et anecdotes, les actes du premier colloque des sociétés d'histoire acadienne de la Nouvelle-Écosse, tenu à l'Université Sainte-Anne en mars 1982, [Pointe-de-l'Église], Université Sainte-Anne (Centre acadien), 1982, 56 p.; [Collectif], Les Cent Lignes de notre américanité, actes de colloque tenu à Moncton du 14 au 16 juin 1984, [préface de Claude Beausoleil], Moncton, Éditions Perce-Neige, 1984, 143 p.; La Réception des œuvres d'Antonine Maillet, actes du colloque international organisé par la Chaire d'études acadiennes les 13, 14 et 15 octobre 1988, publiés par Marguerite Maillet et Judith Hamel, Moncton, Chaire d'études acadiennes, 1989, 339 p.; Vers un aménagement linguistique de l'Acadie du Nouveau-Brunswick, actes du symposium de Moncton, 3, 4 et 5 mai 1990, publiés par Catherine Philipponneau, Moncton, Centre de recherche en linguistique appliquée, Université de Moncton, 1991, 240 p.

${ }^{76}$ Voir la liste des six colloques franco-ontariens dans R. Dionne, La Littérature régionale $[. .$.$] , p. 83, note 117$.

${ }^{77}$ Voir la liste des onze colloques de l'Ouest canadien (1981-1991)
} 
On peut donc dire que, au niveau universitaire, les bases de l'enseignement et de la recherche ont été posées: des professeurs peu nombreux, mais très actifs, ont un bagage d'outils et de connaissances suffisant pour donner un enseignement de bonne qualité. Les inscriptions aux cours du baccalauréat sont nombreuses. Cependant, sauf en Acadie, trop peu d'étudiants des deuxième et troisième cycles orientent leurs recherches dans le champ des trois littératures. Ils craignent sans doute que leurs diplômes soient moins bien considérés si leurs études avancées ont porté sur une littérature régionale, et il se rencontre toujours des professeurs pour confirmer cette crainte ${ }^{78}$. La situation de ces étudiants ne diffère guère de celle que leurs condisciples québécois connurent jusqu'à la fin des années 1960, et même au-delà en quelques lieux, c'est-à-dire avant que la littérature du Québec n'occupât la place qui lui revenait de droit dans les universités de la province. Le temps approche, cependant, où, sous la poussée de l'opinion étudiante devenue publique et devant les exigences des contribuables, les universités locales devront tenir compte de plus en plus des aspirations du milieu qu'elles sont censées desservir dans le respect de ses besoins et de ses valeurs. Nous avons besoin d'une écologie de l'esprit autant que d'une écologie de l'environnement physique.

Les progrès qu'il reste à faire dans le domaine des trois littératures que nous avons considérées dans la présente étude nous semblent dépendre en grande partie de la fierté régionale ou collective que l'on

ibid., pp. 82-83, note 116.

${ }^{78}$ Cette crainte semble courante: "L'étude de phénomènes discrédités est elle-même discréditée.» (Edgar Morin, que nous citons de mémoire.) 
saura insuffler aux élèves du primaire et du secondaire $^{79}$, non seulement dans les classes de langue et de littérature, mais aussi, et de façon tout aussi importante, dans les cours d'histoire et d'autres sciences humaines et sociales. Il ne faut pas oublier, en effet, que si l'écriture se nourrit de l'humain, personnel ou social, dans un contexte économique et politique auquel personne n'échappe, l'exploration des textes qu'elle produit doit s'étendre également à tout ce contexte historique. Ce n'est pas sans raison que l'on a pu remarquer que le développement des études littéraires régionales va de pair avec le développement des études historiques ${ }^{80}$ : c'est dans $^{\prime}$ le creuset de l'histoire que se rencontrent et se fusionnent les diverses expressions de l'humain.

\section{V - Considérations}

L'histoire des trois littératures francophones non québécoises du Canada français au cours des années 1972-1992 est trop courte, ou trop récente sous certains de ses aspects, pour que nous puissions en tirer des conclusions théoriques. Un certain nombre de considérations s'imposent cependant, entre autres sur le rôle de la politique au début de cette histoire et dans la suite.

1. Une attitude politique a provoqué l'émergence des trois littératures. Nous parlons d'attitude, car ce n'est pas surtout la décision du Québec de marcher vers l'indépendance ou vers une souveraineté plus grande qui a déclenché une crise d'identité chez les

\footnotetext{
${ }^{79}$ Depuis 1989 , en Ontario, la littérature franco-ontarienne peut s'enseigner au niveau secondaire.

${ }^{80}$ D'après Guildo Rousseau, cité par $\mathrm{R}$. Dionne, dans la Littérature régionale aux confins de l'histoire et de la géographie, p. 62, notes 12 et 13 .
} 
Canadiens français des autres provinces. Ce fut plutôt, en premier lieu, la façon dont les Québécois ont décidé de ne plus tenir compte des intérêts des minorités francophones du Canada et la manière brutale dont ils ont rejeté la participation de celles-ci à l'établissement d'un nouveau régime canadien; puis ce furent, en second lieu, les prises de position du Québec sur les droits et privilèges des minorités provinciales, entre autres dans le domaine de l'éducation dont le développement et la gestion sont d'une importance primordiale pour la survie des minorités; en troisième lieu, il faut mentionner l'attitude méprisante, quand elle n'est pas indûment paternaliste, de trop de Québécois envers des francophones qu'ils connaissent peu ou prou.

2. La réaction des francophones du Canada a été tellement vive et rapidement organisée qu'elle force à constater qu'existaient déjà des identités régionales bien établies au fond des consciences individuelles. Ces identités avaient été forgées au cours des luttes que les Acadiens, les Franco-Ontariens et les francophones de l'Ouest avaient menées pour leur survivance. Ces minorités avaient, chacune, une histoire et une littérature qui les définissaient déjà; la prise de conscience qu'elles durent en prendre les réconforta au moment où elles se virent forcées de réorienter leurs vies collectives. La prise de parole qui s'ensuivit allait fortifier les volontés et inciter les communautés à récupérer leur passé pour mieux fonder leur spécificité régionale.

3. La rapidité et la vitalité de cette prise de parole ne peuvent pas s'expliquer non plus sans mentionner l'existence d'une tradition et d'une institution littéraires que l'on commence à peine à étudier et qui 
remonte à plus d'un siècle, surtout en Acadie et en Ontario. Au fur et à mesure que l'on étudie les œuvres du passé, on se rend compte que la parole a été prise dès l'occupation du territoire et que ses raisons d'être ressemblent étrangement aux besoins que les rejetés de la tribu québécoise ressentent et expriment aujourd'hui. Les opposants et irritants diffèrent; persiste malgré tout la volonté de vivre librement ce que l'on est par sa naissance et sa culture dans le pays que l'on occupe.

4. Le rôle que les gouvernements fédéral et provinciaux ont joué dans le développement culturel des minorités a permis à ces dernières non seulement de survivre, mais de développer leur culture et leurs institutions. Au point de départ, il y eut la loi des langues officielles adoptée par le gouvernement Trudeau en 1969. Nous avons montré, par l'exemple franco-ontarien, à quel point les ressources financières mises dès lors à la disposition des communautés et des organismes culturels franco-canadiens ont augmenté rapidement à compter de 1970. Cette aide a crû presque continuellement jusqu'en 1992, bien que, en certains domaines, une tendance à la stabilisation, voire à la diminution, semble se dessiner. Deux exemples fédéraux : grâce au programme intitulé «Communautés de langue officielle» (le second en importance budgétaire des dix qui sont administrés par le Secrétariat d'État), les Franco-Manitobains ont reçu les sommes suivantes de 1988-1989 à 1991-1992: 1896901 \$, 1833790 \$, 1804800 \$ et $2055800 \$ \$^{81}$; dans le cadre de son «Programme

\footnotetext{
${ }^{81}$ Renseignements tirés d'un document "préparé et révisé par le bureau régional du Manitoba du Secrétariat d'État le 31 mars 1992»; document transmis par A. Saint-Pierre (Éditions des Plaines, SaintBoniface).
} 
d'initiatives culturelles" (un des quatre à la disposition des organismes culturels francophones), le ministère des Communications a versé, de 1988 à 1992 , 348000 \$ aux Franco-Manitobains, 308000 \$ aux Franco-Albertains et $42000 \$$ aux Fransaskois ${ }^{82}$. La littérature profite, entre autres programmes, de ceux qui viennent en aide à l'édition. Ainsi, de 19851986 à 1991-1992, les Éditions d'Acadie ont reçu 350984 \$ du Conseil des arts du Canada, 362992 \$ du ministère des Communications et, de 1987-1988 à 1991-1992, $153375 \$^{83}$ du Gouvernement du Nouveau-Brunswick.

5. Cette aide gouvernementale diminuant ou disparaissant, on peut penser que la vie serait rendue plus pénible aux francophones du Canada. Ils n'en continueraient pas moins d'exister et d'écrire, car ils ont réussi à se doter d'institutions (écoles, collèges, universités) qui les aideront dans leur lutte perpétuelle de minoritaires, et ils utilisent de plus en plus le concours salutaire de la parole: elle fortifie leur identité en l'exprimant avec plus d'audace et de profondeur et elle réclame avec de plus en plus de force et d'habileté la reconnaissance de leurs droits légitimes de Canadiens à part entière ${ }^{84}$.

\footnotetext{
${ }^{82}$ Renseignements fournis par le ministère des Communications du Canada (région centrale) le 29 avril 1992; ils nous ont été communiqués par A. Saint-Pierre.

${ }^{83}$ Nous devons ces renseignements à James de Finney (Université de Moncton).

${ }^{84}$ Nous remercions les universitaires qui nous ont aidé à rassembler et préciser les renseignements que notre texte contient; ce sont Alain Baudot (Glendon), Fernand Dorais (Laurentienne), James de Finney (Moncton), Pierre Fortier (Glendon), Gaétan Gervais (Laurentienne), Pierre Karch (Glendon), René Le Blanc (Sainte-Anne), Marguerite Maillet (Moncton), Mariel O'Neill-Karch (Toronto), François Paré (Guelph), Jean-Pierre Pichette (Sudbury), Annette Saint-Pierre (SaintBoniface).
} 